\title{
Escolares portadores do alelo a para o polimorfismo rs9939609 do gene FTO apresentam resposta diferente para o índice de massa corporal, após programa de intervenção interdisciplinar?
}

\author{
Schoolchildren allele carrier a for polymorphism rs9939609 (FTO), present different response to \\ body mass index after interdisciplinary intervention program?
}

Priscila Tatiana da Silva ${ }^{1}$, Cézane Priscila Reuter ${ }^{1}$, Kelin Cristina Marques ${ }^{1}$, Pâmela Ferreira Todendi $^{1}$, Elisa Inês Klinger ${ }^{1}$, Miria Suzana Burgos ${ }^{1}$, Andréia Rosane de Moura Valim¹.

${ }^{1}$ Universidade de Santa Cruz do Sul, Santa Cruz do Sul, RS, Brasil.

Submissão: 02/09/2016

Aceite: $23 / 09 / 2016$

RESUMO

pri.pts@hotmail.com

Justificativa e Objetivo: Polimorfismos no gene relacionado à massa gorda e obesidade - FTO (fat mass and obesity associated) têm sido associados com obesidade pelos seus efeitos na homeostase de energia do indivíduo. Assim, busca-se verificar se escolares portadores do alelo de risco para obesidade (A), do polimorfismo rs9939609, do gene FTO, respondem diferentemente a um programa de intervenção com exercício físico e orientação nutricional, em comparação aos escolares que não possuem este alelo, com relação ao Índice de Massa Corporal (IMC). Métodos: estudo quase-experimental, realizado com 46 escolares, distribuídos, igualmente, em grupos experimental e controle, da zona rural do município de Santa Cruz do Sul-RS. A variante A/T, do polimorfismo rs9939609 do gene FTO, foi genotipada utilizando-se o sistemaTaqMan ${ }^{\mathrm{TM}}$, em equipamento automatizado StepOne Plus. O programa interdisciplinar teve duração de 4 meses, sendo realizado 3 vezes por semana, no turno contrário ao das aulas. A duração de cada foi de 1 hora e 30 minutos. Resultados: no grupo experimental, um escolar com genótipo TT que apresentava sobrepeso, passou a ter IMC normal após o programa. Para os genótipos AT + AA, os escolares mantiveram a classificação (obesidade), sendo que um escolar que apresentava sobrepeso, passou a apresentar obesidade, após o término da intervenção. A análise dos valores de IMC, antes e após a intervenção, revelou que não houve diferença significativa, tanto no grupo experimental $(p=0,088)$, quanto no controle $(p=0,964)$. Conclusão: independente do genótipo, os escolares responderam de forma semelhante ao programa interdisciplinar com exercícios físicos e orientação nutricional.

DESCRITORES: Obesidade. Polimorfismo. Escolares.

\begin{abstract}
Justificative e Objective: Polymorphisms in the gene related to fat mass and obesity - FTO has been associated with obesity by its effects in the individual's energy homeostasis. Thus, we seek to verify if the children with risk allele (A) for obesity polymorphism rs9939609 in the FTO gene, respond differently to an intervention program with physical and nutritional counseling exercise, compared to students who do not have this allele, with relation to body mass index. Methods: A quasi-experimental study with 46 students, distributed also in experimental and control group, the rural municipality of Santa Cruz do Sul-RS. The variant A/T polymorphism rs9939609, the FTO gene was genotyped using the system TaqMan in Step One Plus automated equipment. The interdisciplinary program lasted four months, being held 3 times per week, in turn contrary to the classes. The duration of each session was 1 hour and 30 minutes. Results: In the experimental group, a school with TT genotype were overweight, went on to have a normalBMI after of the
\end{abstract}


program. For AT + AA genotypes, the school kept the score (obesity), and a school who were overweight before the the intervention began to show obesity after termination thereof. The analysis of BMI values before and after the intervention, revealed that there was no significant difference in both the experimental group $(p=0.088)$ and in the control $(p=0.964)$. Conclusion: the schoolchildren, regardless of genotype, responded similarly to the interdisciplinary program with exercise and nutritional guidance.

KEYWORDS: Obesity. Genetics. School.

\section{INTRODUÇÃO}

A obesidade é uma condição multifatorial, influenciada por fatores comportamentais, psicológicos e genéticos. ${ }^{1,2}$ Dados epidemiológicos evidenciam que a prevalência de sobrepeso e obesidade em crianças e adolescentes teve aumento de $47 \%$ de 1980 a $2013 .{ }^{3}$ No Brasil, resultados de estudos desenvolvidos em Campinas-SP e Florianópolis-SC, envolvendo crianças e adolescentes de 7 a 14 anos apontou prevalência de obesidade entre $21,1 \%$ a $31,5 \%$, respectivamente, fato que implica em perspectivas de saúde cada vez mais preocupantes. ${ }^{4,5}$ Por outro lado, avanços científicos recentes tem demonstrado que o componente genético possui uma participação relevante na ocorrência da obesidade, estando relacionada com a presença de polimorfismos. ${ }^{6}$

Deste modo, diversos polimorfismos estão sendo investigados e considerados importantes marcadores da obesidade. Além disso, a aproximação de um polimorfismo de um locus que origina determinada condição ou doença, tende a fazer com que este passe a incorporar, também, o alelo causador da doença. ${ }^{7,8}$ Dessa forma, polimorfismos no gene relacionado à massa gorda e obesidade - FTO (fat mass and obesity associated) tem sido explorados pela sua associação com a obesidade e sua possível associação com efeitos na homeostase de energia, que podem afetar a adipogênese, a função mitocondrial, a lipogênese e o músculo esquelético. ${ }^{6}$

Um estudo anterior desenvolvido no múnicipio de Santa Cruz do Sul-RS, com 420 escolares de 7 a 17 anos, avaliou a relação entre a massa de gordura rs9939609 do polimorfismo FTO com aptidão cardiorespiratória (APCR) e sobrepeso/obesidade. Os resultados identificaram que estudantes com baixos niveis de APCR e pertecentes ao genótipo AA possuem maior risco para desenvolver sobrepeso e obesidade. Complementarmente, o estudo identificou que o polimorfismo rs9939609(FTO) demonstrou relação com a obesidade e APCR na população pesquisada. ${ }^{9}$ Relação similar foi encontrada em estudo desenvolvido em Shanghai-China em que escolares do genótipo AA ou AT apresentaram IMC significativamente maior em comparação a escolares do genótipo TT $(\mathrm{p}<0,044) .^{10}$

Neste contexto, compreende-se que as características de um indivíduo dependem de um conjunto de informações genéticas herdadas (genótipo) e das interações destas com as ações do meio ambiente (fenótipo). ${ }^{11}$ Portanto, conhecer os fatores de riscos genéticos associados com a 
obesidade infanto-juvenil pode ser útil na projeção de estratégias de prevenção de saúde para esta população. $^{12}$

Deste modo, o presente estudo objetiva verificar se escolares portadores do alelo de risco para obesidade (A), do polimorfismo rs9939609, no gene $F T O$, respondem diferente a um programa de intervenção interdisciplinar com exercício físico e orientação nutricional, em comparação aos escolares que não possuem este alelo, com relação ao índice de massa corporal.

\section{MÉTODOS}

O presente estudo, de caráter quase-experimental, envolveu 46 escolares, pertencente a uma população predominantemente caucasiana (23 para o grupo experimental e 23 para o grupo controle), com idade entre seis e 16 anos, do meio rural de Santa Cruz do Sul-RS. ${ }^{9}$ Todos os escolares apresentavam sobrepeso ou obesidade, características semelhantes em relação à idade, aspectos culturais e indicadores antropométricos. O cálculo do tamanho amostral foi realizado no programa $G^{*}$ Power, sendo sugerido 12 sujeitos, no mínimo, para cada grupo, considerando um poder de teste de 0,8 , efeito de 0,30 e nível de significância de $95 \%$. ${ }^{13} \mathrm{O}$ estudo foi previamente submetido e aprovado pelo Comitê de Ética em Pesquisa com Seres Humanos da Universidade de Santa Cruz do Sul (UNISC), sob protocolo 357.403/2013. Todos os pais ou responsáveis pelos escolares assinaram um Termo de Consentimento Livre e Esclarecido, autorizando a participação na pesquisa.

O IMC foi avaliado considerando as medidas de peso e estatura. Após aplicação da fórmula $\left(\mathrm{IMC}=\mathrm{peso} /(\text { estatura })^{2}\right.$ ), os valores foram classificados de acordo com os pontos de corte estabelecidos para crianças e adolescentes, propostos pelo Centers for Disease Control and Prevention/National Center for Health Statistics (CDC/NCHS), considerando baixo peso (<p5), normal $(\geq \mathrm{p} 5$ e $<\mathrm{p} 85)$, sobrepeso $(\mathrm{p} \geq 85$ e $<\mathrm{p} 95)$ e obesidade $(\geq \mathrm{p} 95)$, de acordo com o sexo e a idade do escolar. ${ }^{14}$

A extração do DNA foi realizada através do método de Salting out, com amostras de sangue total, anticoaguladas com EDTA. ${ }^{15}$ Foi empregada a técnica de Reação em cadeia da polimerase em tempo real (qPCR) para a genotipagem. As variantes A/T, do polimorfismo rs9939609, do gene FTO, foi investigada utilizando-se sondas do tipo TaqMan, em equipamento automatizado StepOne Plus (Applied Biosystems, Foster City, CA, USA).

O programa interdisciplinar teve duração de 4 meses, sendo realizado 3 vezes por semana, no turno contrário ao das aulas. Cada sessão consistia de atividades com duração de 1 hora e 30 minutos. Os participantes (grupo experimental) realizaram aquecimento (10 minutos), exercícios aeróbicos (50 minutos) e com caráter lúdico (20 minutos). Todos os exercícios foram realizados 
utilizando monitor cardíaco da marca Polar, modelo FT1 (Polar, Dinamarca). Foram realizados, também, exercícios para reeducação postural e atividades de orientação nutricional. Para os exercícios de reeducação postural os escolares foram submetidos a realização de exercícios dinâmicos e isométricos de alongamentos e fortalecimento muscular, as atividades incluíram o uso de materiais diversificados e específicos como bolas suíças (Pilates), faixas elásticas e flutuadores para piscina (espaguetes e pranchas). Inicialmente, os exercícios foram realizados em serie única, com 10-15 exercícios mantidos por 20 segundos e intercalados com 15 segundos de intervalo. O grau de exigência dos exercícios foi modificado e aprimorado através da manutenção do tempo de execução do mesmo, conforme evolução mensal dos alunos. Para as atividades de orientação nutricional, foi enfatizada a abordagem da pirâmide alimentar, a comparação de alimentos e seus valores nutritivos, palestras e documentários sobre o uso de açúcar, sal, lipídios e a prevenção de doenças futuras, bem como aulas de culinária e elaboração de folder e cartazes educativos junto aos alunos.

Os dados foram analisados no programa estatístico SPSS v. 20.0 (IBM, Armonk, USA). A comparação da frequência da classificação do Índice de Massa Corporal (IMC), antes e após o programa interdisciplinar, foi realizada através do teste exato de Fisher. Para os dados contínuos, utilizou-se a mediana e o intervalo interquartílico, bem como o teste de Wilcoxon para comparação entre os genótipos. Foram consideradas significativas as diferenças para $\mathrm{p}<0,05$.

\section{RESULTADOS}

Os resultados demonstram que, entre o grupo experimental, um escolar com genótipo TT apresentava sobrepeso, antes do início da intervenção, sendo que passou a ter IMC normal após a realização do programa. Para os genótipos AT + AA, os escolares mantiveram a classificação (obesidade), sendo que um escolar que apresentava sobrepeso, antes do início da intervenção, também passou a apresentar obesidade, após o término da mesma (Tabela 1).

Tabela 1 - Classificação do IMC, antes e após a realização da intervenção interdisciplinar

\begin{tabular}{|c|c|c|c|c|c|c|}
\hline$Y$ & & & \multicolumn{3}{|c|}{ Após a intervenção } & \multirow{2}{*}{$\mathrm{P}$} \\
\hline & & Antes da intervenção & Normal & Sobrepeso & Obesidade & \\
\hline \multirow{4}{*}{$\begin{array}{l}\text { Controle } \\
(\mathrm{N}=23)\end{array}$} & \multirow{2}{*}{$\mathrm{TT}(\mathrm{N}=11)$} & Sobrepeso & 2 & 3 & 0 & \multirow{4}{*}{0,061} \\
\hline & & Obesidade & 0 & 2 & 4 & \\
\hline & \multirow{2}{*}{$\begin{array}{c}\mathrm{AT}+\mathrm{AA} \\
(\mathrm{N}=12)\end{array}$} & Sobrepeso & - & 6 & 0 & \\
\hline & & Obesidade & - & 2 & 4 & \\
\hline \multirow{4}{*}{$\begin{array}{l}\text { Experimental } \\
\qquad(\mathrm{N}=23)\end{array}$} & \multirow{2}{*}{$\mathrm{TT}(\mathrm{N}=7)$} & Sobrepeso & 1 & 2 & 0 & \multirow{4}{*}{0,003} \\
\hline & & Obesidade & 0 & 0 & 4 & \\
\hline & \multirow{2}{*}{$\begin{array}{c}\mathrm{AT}+\mathrm{AA} \\
(\mathrm{N}=16)\end{array}$} & Sobrepeso & - & 4 & 1 & \\
\hline & & Obesidade & - & 0 & 11 & \\
\hline
\end{tabular}


Teste exato de Fisher; genótipos para o polimorfismo rs9939609 (FTO), considerando o alelo A de risco para obesidade (genótipos AT + AA).

Analisando os valores de IMC, antes e após o programa de intervenção, observa-se que não houve diferença significativa, tanto no grupo experimental $(p=0,088)$, quanto no controle $(p=0,964)$ (Tabela 2).

Tabela 2 - IMC, antes e após a realização da intervenção interdisciplinar

\begin{tabular}{ccccc}
\hline & & Antes da intervenção & Após a intervenção & P \\
\hline \multirow{2}{*}{$\begin{array}{c}\text { Controle } \\
(\mathrm{N}=23)\end{array}$} & $\mathrm{TT}(\mathrm{N}=11)$ & $24,5(22,9-28,2)$ & $24,5(22,3-28,4)$ & 0,964 \\
\cline { 2 - 4 } & $\mathrm{AT}+\mathrm{AA}(\mathrm{N}=12)$ & $24,3(20,1-27,6)$ & $23,5(20,6-27,0)$ & \\
\cline { 1 - 4 } $\begin{array}{c}\text { Experimental } \\
(\mathrm{N}=23)\end{array}$ & $\mathrm{TT}(\mathrm{N}=7)$ & $27,2(19,0-29,1)$ & $27,9(18,5-31,0)$ & 0,088 \\
\cline { 2 - 4 } & $\mathrm{AT}+\mathrm{AA}(\mathrm{N}=16)$ & $24,2(23,0-25,8)$ & $24,8(22,7-28,0)$ & \\
\hline
\end{tabular}

Valores expressos em mediana (intervalo interquartílico); Teste de Wilcoxon; genótipos para o polimorfismo rs9939609 (FTO), considerando o alelo A de risco para obesidade (genótipos AT + AA).

\section{DISCUSSÃO}

Os resultados do presente estudo demonstraram que o programa de intervenção com exercícios foi capaz de propiciar mudanças no padrão de biótipo de um escolar do genótipo TT, que passou da zona de sobrepeso para a zona de classificação de IMC normal. Contudo, resultados similares não foram encontrados para o grupo de genótipos AT+ AA. Estes resultados podem ser explicados pela ação dominante do genótipo AA, que corresponde a cerca de $1 \%$ da hereditariedade genética. De acordo com Albuquerque, Nóbrega e Manco, a presença de cada alelo A, do polimorfismo rs9939609, pode aumentar o peso corporal em $1,5 \mathrm{~kg}$ no adulto, tendo efeitos semelhantes em crianças e adolescentes. Em seu estudo, os autores constaram que para a presença de cada alelo A do polimorfismo FTO rs9939609, o efeito obtido foi ganho de aproximadamente $0,6 \mathrm{~kg} / \mathrm{m}^{2}$ no IMC, $1,2 \mathrm{~cm}$ de circunfêrencia da cintura e $1,7 \mathrm{~kg}$ de peso total. ${ }^{12}$

No Reino Unido, um estudo realizado a partir de 3 coortes com 38.789 participantes apontou que para cada estudo, indivíduos com dois alelos A tinham um IMC maior em comparação a indivíduos com dois alelos diferentes. Resultados similares foram encontrados em estudo realizado em Taiwan-China, em que foram avaliados 594 indivíduos jovens obesos, no grupo experimental. Para essa amostra, foram genotipados 19 polimorfismos de nucleotídeo único (SNPs), sendo que entre os 19 SNPs, o rs9939609 foi fortemente associado à obesidade. A razão de chances para a

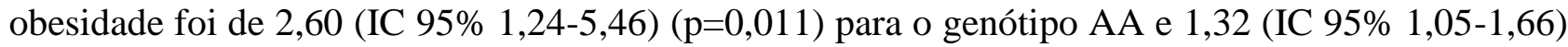
( $\mathrm{p}=0,018)$ para o genótipo AT, em comparação com o genótipo TT. A variação genética no gene FTO rs9939609, deste modo, foi fortemente associada com a obesidade e IMC nessa amostra da população chinesa. ${ }^{16}$ 
Em nosso estudo, constatou-se que para os genótipos AT + AA, os escolares mantiveram a classificação de obesidade, sendo que um escolar que apresentava sobrepeso, antes do início da intervenção, passou a apresentar, também, obesidade, após o término da intervenção. Esse dado corrobora com resultados encontrados por Reinehr et al., que investigou 346 crianças europeias, com média de idade de 10,6 \pm 2,6 anos, com sobrepeso, e constatou que para o alelo de risco, no gene $F T O$, não foi observada a redução de IMC durante a intervenção de estilo de vida, tendo, inclusive os escolares apresentado uma recuperação de peso um ano após o final da intervenção; porém, para crianças com genótipo sem predisposição ao risco da obesidade, houve uma diminuição nos valores de IMC. ${ }^{17}$

Outro ponto observado em nosso estudo foi que os valores de IMC, antes e após o programa de intervenção, não apresentaram diferenças significativas, tanto no grupo experimental ( $\mathrm{p}=0,088$ ), quanto no controle $(\mathrm{p}=0,964)$. De forma semelhante, alguns estudos não encontraram qualquer resposta relacionadas ao gene $F T O$ e programas de intervenção com exercícios físicos e orientação nutricional, o que sugere que outras variáveis genéticas influenciam nas ações que envolvem esses genes. ${ }^{17,18}$ Entre os fatores que supostamente afetam as ações propostas pelo polimorfismo rs9939609 FTO, dados de um estudo realizado em dez cidades da Europa, apontam que a ingestão dietética possui influência direta nesta variável, estando relacionada a massa de gordura corporal e obesidade. O estudo realizado com 652 adolescentes constatou que ocorrem interações significativas associadas ao polimorfismo no gene $F T O$ e ingestão de gordura $(\mathrm{p}<0,05)$, sendo que adolescentes com consumo de gordura entre 30\% e 35\% da energia $(n=190)$ apresentaram elevação de gordura corporal de $1,9 \%$ a mais por alelo de risco associada ao polimorfismo rs9939609 (IC 95\%: $0,39,3,33 ; \mathrm{p}<0,05)$. Além disso, escolares em que a ingestão de gordura foi superior a $35 \%$ $(n=259)$, o risco elevou-se para 2,8\% a mais por alelo de risco. A associação entre gordura corporal e o polimorfismo rs9939609 foi evidenciada ainda pelo fato de que em adolescentes com ingestão de gordura inferior a 30\% $(\mathrm{n}=203)$, o alelo de risco no polimorfismo rs9939609 não esteve associado com índices de adiposidade. Deste modo, os resultados sugerem que o efeito prejudicial do polimorfismo rs9939609 FTO é ampliado em adolescente que possuem elevado consumo de gorduras e da mesma forma o consumo reduzido de gordura ( $<30 \%$ de energia) pode atenuar a predisposição genética para a obesidade em portadores do alelo de risco. ${ }^{19} \mathrm{Em}$ contrapartida, um estudo realizado na Alemanha, com 75 crianças e adolescentes, com excesso de peso, buscou investigar o impacto das variantes do gene FTO (rs17817449, rs1421085, rs9939609) em crianças obesas antes e após um programa de intervenção de 6 meses, e constatou que para toda a população do estudo, houve melhora significativa nos valores de $\operatorname{IMC}(0,10 \pm 0,17, \mathrm{p}<0,001)$ e porcentagem de gordura corporal $(0,09 \pm 0,23, \mathrm{p}=0,005)$, sem diferença entre grupos. Os autores concluem que as 
variantes dos genes $F T O$ são comuns em crianças obesas, mas não influenciam na composição corporal, antes ou durante o programa de intervenção. ${ }^{18}$

Ressaltamos que esse estudo é pioneiro no Brasil, em investigar se portadores de alelo de risco para a obesidade respondem de forma diferente a um programa interdisciplinar com exercícios físicos em escolares com excesso de peso, com relação ao índice de massa corporal. Porém, citamos algumas limitações do estudo, como o tamanho amostral pequeno e a não inclusão de aspectos étnicos nas análises. De acordo com Lima, Glaner e Taylor e Albuquerque, Nóbrega e Manco, as características étnicas podem impactar nos resultados, sendo que indivíduos caucasianos, portadores do homozigotos A para a variante FTO rs9939609, possuem maior propensão ao acúmulo de gordura corporal excessivo; em sujeitos não-caucasianos, a influência deste alelo foi menos expressiva. ${ }^{11,12}$ Dessa forma, sugerimos a realização de estudos experimentais com um número maior de sujeitos, bem como analisar o efeito de múltiplas variáveis sobre a obesidade, uma vez que esta condição é de origem multifatorial. Os resultados do presente estudo demonstram que, para o grupo experimental, um escolar com genótipo TT com sobrepeso passou a apresentar classificação de IMC normal após a realização do programa. Para os genótipos AT + AA, os escolares mantiveram a classificação (obesidade). Porém, nosso estudo não encontrou diferença significativa, tanto no grupo experimental, quanto no controle, no que se refere aos valores de IMC, antes e após o programa de intervenção.

\section{REFERÊNCIAS}

1. Melzer MRTF, Magrini IM, Domene SMA, et al. Factors associated with abdominal obesity in children. Rev Paul Pediatr 2015;33(4):437-44.

2. Hebebrand J, Hinney A, Knoll N, et al. Molecular genetic aspects of weight regulation. Dtsch Arztebl Int 2013;110(19):338-44.

3. Snethen JA, Broome ME, Treisman P, et al. Effective weight loss for children: a meta-analysis of intervention studies 2002-2015. Worldviews Evid Based Nurs 2016: 13(4):294-302.

4. Rossi CE, Vasconcelos FAG. Relationship between birth weight and overweight/obesity among students in Florianópolis, Santa Catarina, Brazil: a retrospective cohort study. Sao Paulo Med J 2014: 132(5): 273-81.

5. Castilho SD, Nucci LB, Hansen LO, et al. Prevalence of weight excess according to age group in students from Campinas, SP, Brazil. Rev Paul Pediatr 2014; 32(2): 200-6.

6. Apalasamy YD, Ming MF, Rampal S, et al. Genetic association of SNPs in the FTO gene and predisposition to obesity in Malaysian Malays. Braz J Med Biol Res 2012; 45(12):1119-26. 
7. Pierce BA. Genética: um enfoque conceitual. 3. ed. Rio de Janeiro: Guanabara Koogan, 2011.

8. Passarge E. Genética texto e atlas. 3. ed. Porto Alegre: Artmed, 2011.

9. Reuter CP, Valim ARM, Gaya AR, et al. FTO polymorphism, cardiorespiratory fitness, and obesity in Brazilian youth. Am J Hum Biol 2016;28(3):381-6.

10. Zou ZC, Mao J, Shi YY, et al. Effect of exercise combined with dietary intervention on obese children and adolescents associated with FTO rs 993909 polymorphism. Eur Rev Med Pharmacol Sci 2015:19(23):4569-75.

11. Lima WA, Glaner MF, Taylor AP. Fenótipo da gordura, fatores associados e o polimorfismo rs9939609 do gene FTO. Rev Bras Cineantrop Desemp Humano 2010;12(2):164-72.

12. Albuquerque D, Nóbrega C, Manco L. Association of FTO polymorphisms with obesity and obesity-related outcomes in Portuguese children. PloS One 2013;8:543-70.

13. Faul F, Erdfelder E, Lang AG, et al. G*Power 3: A flexible statistical power analysis program for the social, behavioral, and biomedical sciences. Behav Res Methods 2007;39:175-91.

14. CDC. Centers for Disease Control and Prevention/National Center for Health Statistics. CDC Growth Charts: United States. 2000. Disponível em: 〈http://www.cdc.gov/growthcharts〉. Acesso em: 12 dez. 2015.

15. Miller SA, Dykes DD, Polesky HF. A simple salting out procedure for extracting DNA from human nucleated cells. Nucleic Acids Research 1988;16(3):1215.

16. Chang YC, Liu PH, Lee WJ, et al. Common Variation in the Fat Mass and Obesity-Associated (FTO) Gene Confers Risk of Obesity and Modulates BMI in the Chinese Population. Diabetes 2008;57(8):2245-52.

17. Reinehr T, Wolters B, Roth CL, et al. FTO Gene: Association to weight regain after lifestyle intervention in overweight children. Horm Res Paediatr 2014;81(6):391-6.

18. Schum J, Blumenstock G, Weber K, et al. Variants of the FTO gene in obese children and their impact on body composition and metabolism before and after lifestyle intervention. Exp Clin Endocrinol Diabetes 2012;120(3):128-31.

19. Labayen I, Ruiz JR, Huybrechts I, et al. Dietary fat intake modifies the influence of the FTO rs9939609 polymorphism on adiposity in adolescents: The HELENA cross sectional study. Nutr Metab Cardiovasc Dis 2016: (16)1-7. 